Research Article

\title{
Research on the Identification of Key Nodes in the Process of WeChat Epidemic Information Dissemination: A Supernetwork Perspective
}

\author{
Peng Wu iDd Di Zhao \\ Institute of Management Science and Engineering, Henan University, Kaifeng 475004, China \\ Correspondence should be addressed to Peng Wu; akasfd@126.com
}

Received 24 June 2020; Accepted 25 July 2020; Published 27 August 2020

Guest Editor: Shaohui Wang

Copyright (c) 2020 Peng Wu and Di Zhao. This is an open access article distributed under the Creative Commons Attribution License, which permits unrestricted use, distribution, and reproduction in any medium, provided the original work is properly cited.

\begin{abstract}
It is of great significance to comprehensively and reasonably identify the key nodes in the WeChat epidemic information dissemination system. First, the recognition results can be used to guide the spread of epidemic-related information in WeChat, such as accelerating the spread of valuable information or monitoring the spread of rumors. Secondly, the analysis of key nodes helps us understand the evolution of the epidemic information dissemination network in WeChat, and the analysis of key nodes also helps us understand the modes and methods of epidemic-related information dissemination in WeChat. Finally, the results of these studies may be generalized to other fields of social life. This paper analyzes the composition of and relationship between epidemic-related information dissemination systems in WeChat and proposes a Supernetwork model for WeChat epidemic information dissemination on this basis. In this study, a comprehensive identification method of key nodes of the WeChat epidemic information dissemination system under the Supernetwork vision was constructed, and the method is analyzed and verified through examples in this paper.
\end{abstract}

\section{Introduction}

With the rapid development of information technology and the increasingly complex social system, the current information dissemination related to the epidemic situation is also becoming more and more rapid. If the spread of relevant public opinion or rumors in a network cannot be promptly understood and effectively guided and managed, the spread of its outbreak will have catastrophic consequences. Therefore, it is necessary to conduct research and analysis on the spread of information related to the epidemic situation [1].

Tencent released the "2019 WeChat Annual Data Report" in January 2020. The report stated that in 2019, the number of WeChat monthly active accounts exceeded 11 billion [2]. With such a huge monthly active user scale, WeChat's role in the spread of epidemic information cannot be underestimated.
After the outbreak, WeChat, as a highly active and highly connected medium, has become an important channel for the public to understand and participate in the epidemic. Scholar Liu [3] used the framework analysis theory to systematically analyze the top $200 \mathrm{WeChat}$ posts using the Qingbo Index over a period of time and found that the top posts on WeChat during the epidemic mainly showed the following characteristics. In terms of media report types, both self-media and traditional media commonly guide the network public opinion pattern. In terms of the evolution of public opinion, online public opinion events frequently occur and advance in waves. In the news framework, the response framework and the emotional framework have the highest public acceptance and recognition. Through the above research, Liu proposed that the guidance of public opinion on the epidemic should strengthen the "strength" of rational dissemination, pay attention to the "temperature" of humanistic care, and improve the "validity" of public 
opinion guidance. Based on a full-sample empirical analysis of 2966 official WeChat public account articles from 42 "double first-class" universities, Deng et al. [4] found that article topics, information sources, and emotional tendencies have a significant impact on communication effects. Public opinion propaganda with a richer connotation is more popular. The original works of universities' WeChat public accounts are more influential. Public opinion propaganda with obvious "positive" or "negative" sentimental tendencies has a significant impact on the communication effect but in different ways. Readers who read short and concise articles are more likely to keep reading until the end of the article and like the article. Chen [5] found that rumors showed a rapid growth trend in the early stages of the outbreak. The formation and spread of rumors mainly occurs on WeChat and Weibo, and the spread of rumors is directly related to the degree of government information disclosure. Due to the strong relationship and semienclosed space feature of WeChat, WeChat rumors are mainly spread through public accounts, group chats, private chats, and circles of friends. They have both interpersonal and group transmission characteristics and are extremely infectious. As the number of confirmed diagnoses across the country began to decline, and some cities began to resume work, the government continued to disclose information about the epidemic in an open and transparent manner, and related rumors began to decline. During this period, the government responded quickly to rumors, and there gradually became fewer "unverified" rumors. At the same time, the spread of strong relationships in WeChat groups allowed the public to receive a large amount of factual splicing from relatives and friends in different spaces, which eliminated rumors to a great extent. The ambiguity in the communication model improved the public's judgment, and the rumors lost their foundation for spreading. Wan et al. [6] used the case study method to establish the status quo of the "fitness antiepidemic tweets" pushed by the 117 WeChat subscription accounts they followed and found that their main features are the time and frequency of article posting, which are consistent with the changes in the number of confirmed cases, and the overall national prevention and control deployment. The online dissemination of content is comprehensive, and the amount of information is gradually decreasing with the effective relief of the epidemic. By analyzing popular words in WeChat, Lu and Zhang [7] found that the development of the epidemic had a certain pattern. First of all, people paid attention to which pathogen the disease comes from, the location of the outbreak, and what symptoms appear. Then, the public began to pay attention to the source of pathogens, the behavior and self-protection of medical staff, and the needs of daily life. On the other hand, people paid more attention to the epidemic situation in other places and the results of local control. Through the analysis of public concern, we reviewed the development trend of the epidemic, which will set an example for future epidemics. It is believed that public health authorities will rely more on these social media to monitor the development of epidemics or pandemics in the future. Judging from the existing related results, there are few studies in this field, and the use of Supernetwork theory to study the spread of epidemic information in WeChat is still rare.

This article studies and analyzes the composition and relationship between the epidemic-related information dissemination systems in WeChat. On this basis, three types of active and representative elements are established, namely, ordinary WeChat users, WeChat groups, and WeChat public accounts. The three epidemic information dissemination network relationships are combined and integrated into a Supernetwork.

\section{Supernetwork Theory and WeChat Supernetwork Model Construction}

Scholars previously used the term "Supernetwork" in the fields of computer systems, genetics, and transportation systems to generally refer to complex systems with complicated nodes, complicated relationships, and networks within them. In the American scholar Nagurney's work on interwoven networks, the network that is higher than and surpasses the existing network is referred to as the "Supernetwork," which helps to clearly define this term $[8,9]$. Chinese scholars, Academician Wang Zhongtuo and Professor Wang Zhiping, further proposed the relevant characteristics of the Supernetwork. They believe that the Supernetwork has one or more of the following characteristics: (1) The network contains the network, or it can be said that the network is nested in the network. (2) It has the characteristics of multiple layers, and there are connections within and between layers. For example, the protocol of the information network is multilayered, and the transportation network has a management layer, a business layer, and a physical layer, which are also multilayered. (3) It has the characteristics of multiple levels, and there is a connection between these levels. For example, the information network of an enterprise has multiple levels such as headquarters, companies, and departments. (4) Its flow can have multidimensional characteristics. For example, aviation, roads, water transportation, and railways all have both freight and passenger transportation. (5) It has the characteristics of multiple attributes or multiple criteria. For example, the transportation network needs to consider factors such as cost, safety, time, and comfort at the same time, and when traveling in a city, there are not only travel modes (such as self-driving, public transportation, or walking) but also route options. (6) It has the characteristics of congestion. For example, the transportation network has the problem of congestion, as does the information network. (7) Sometimes, there is a conflict between individual optimization and global optimization, which requires coordination [9].

Recent research on Supernetworks can be divided into three directions: based on variational inequalities [10-12], the work mainly intends to transform the multilayered, multistandard Supernetwork balance model into an optimization problem and then use evolutionary variation inequality to solve the problem [9]. The system science [13-16] research studies the Supernetwork as a whole as well as individual aspects, including the study of the relationship between the network and the network in the Supernetwork, 
the study of the network using the relationship between the outside and the network, and the study of overall performance. The modeling work of this article is mainly inspired and helped by some research results in this area. Research based on hypergraphs [17] is considered to be another more important research direction. It is mostly used to solve the problem of different textures between edges and nodes in network research. Hypergraphs are used to describe the system and study the statistical characteristics of the network. The research on Supernetworks has developed rapidly, and its application fields are also relatively wide, such as knowledge management, supply chains, transportation, financial management, advertising, e-commerce, biology, and organization and cooperation [18]. However, Supernetwork theory and methods are rarely applied to the field of WeChat epidemic information dissemination.

WeChat public accounts, common WeChat users, and WeChat groups are the main outlets through which epidemic information is spread in WeChat. The public account mainly disseminates information through the weak relationship between the public account and the fans. The public account and the fans form a star network. Relatively speaking, the communication speed is fast and the spread is wide, but the audience trusts the public account to release information, though this is not the most trusted source of information.

The ordinary users of WeChat mainly spread information through the strong relationship between ordinary users' friends. The information publisher and information receiver in the circle of friends also form a star network. Because of the relationships between acquaintances, the information posted in the circle of friends is related to the circle of friends. Members tend to believe this information more, but the information in the circle of friends is limited by the border barriers of the circle of friends. On the whole, there is a large gap between the speed of propagation and the range of communication compared with the public account.

The WeChat group has special properties. The WeChat group can take into account both strong and weak relationships for propagation; that is, members in the WeChat group may not be known or trusted, and members of the WeChat group may or may not be influential. This can break the barriers of the circle of friends based on strong relationships, so that the spread of epidemic-related information is wider and faster. The degree to which the recipient of the information believes the information is based on these factors.

In summary, this article integrates these three elements into a "Supernetwork" of WeChat epidemic information dissemination systems based on the association between the public accounts, common WeChat users and WeChat groups, referred to as the WeChat epidemic information dissemination Supernetwork.

\subsection{Supernetwork Modeling of WeChat Epidemic Information} Dissemination. From the previous analysis, we can see that there are three types of epidemic information dissemination networks in Figure 1:
(1) The P-P common WeChat user relationship network's nodes are common WeChat users. The network can be expressed as $G_{p}=\left(P, E_{\mathrm{P}-\mathrm{P}}\right)$, where $P=\left\{p_{1}, p_{2}, \ldots, p_{b}\right\}$ is a set of common WeChat user relationship network members; $E_{\mathrm{P}-\mathrm{P}}=\left\{\left(p_{i}, p_{j}\right) \mid p_{i}\right.$, $\left.p_{j} \in P\right\}$ is a set of edges; and $\left(p_{i}, p_{j}\right)$ is a common WeChat user. There is an association between $p_{i}$ and $p_{j}$

(2) The M-M WeChat group network is the network constructed by the WeChat group. The nodes are WeChat groups, and the edges represent the association between WeChat groups. Its model is $G_{m}=\left(M, E_{\mathrm{M}-\mathrm{M}}\right) ; M=\left\{m_{1}, m_{2}, \ldots, m_{a}\right\}$ is a collection of WeChat groups; $E_{\mathrm{M}-\mathrm{M}}=\left\{\left(m_{i}, m_{j}\right) \mid m_{i}\right.$, $\left.m_{j} \in M\right\}$ is a collection of association relationships between WeChat groups; and edge $\left(m_{i}, m_{j}\right)$ indicates that there is an association between WeChat groups $m_{i}$ and $m_{j}$.

(3) The I-I WeChat public account network is formed between public accounts. The node is a WeChat public account. The network can be described as $G_{i}=\left(I, E_{\mathrm{I}-\mathrm{I}}\right) ; I=\left\{i_{1}, i_{2}, \ldots, i_{c}\right\}$ is a collection of public accounts; and $E_{\mathrm{I}-\mathrm{I}}=\left\{\left(i_{i}, i_{j}\right) \mid i_{i}, i_{j} \in I\right\}$ is a collection of associations between public accounts. $\left(i_{i}, i_{j}\right)$ indicates that there is an association between $i_{i}$ and $i_{j}$.

The following mapping relationships exist between the three networks:

(1) The mapping between WeChat general users and WeChat public accounts refers to which ordinary WeChat users pay attention to which public accounts and which common WeChat users follow which public accounts.

(2) The mapping between WeChat groups and public accounts refers to which public accounts are associated with which WeChat groups.

(3) The mapping between ordinary WeChat users and WeChat groups refers to which ordinary WeChat users join or are added to which WeChat groups, which WeChat groups are associated, and which WeChat groups include which ordinary WeChat users.

According to the mapping relationship described above, a mapping relationship can be established between $G_{i}, G_{p}, G_{m}$ nodes so that they are integrated together to construct a Supernetwork with three types of nodes. The specific approach is based on the three networks that already exist, and the mapping relationship between different types of nodes is also added as an edge, thereby integrating three different types of networks together. This process can be expressed as follows.

Let $p_{i} \in P, i_{j} \in I$, and $m_{l} \in M$, respectively, represent any node of the WeChat general user association network, WeChat public account network, and WeChat group association network. The Boolean variables $\phi\left(p_{i}, i_{j}\right), \varphi\left(p_{i}, m_{l}\right)$, and $\gamma\left(i_{j}, m_{l}\right)$ indicate whether the different types of nodes 


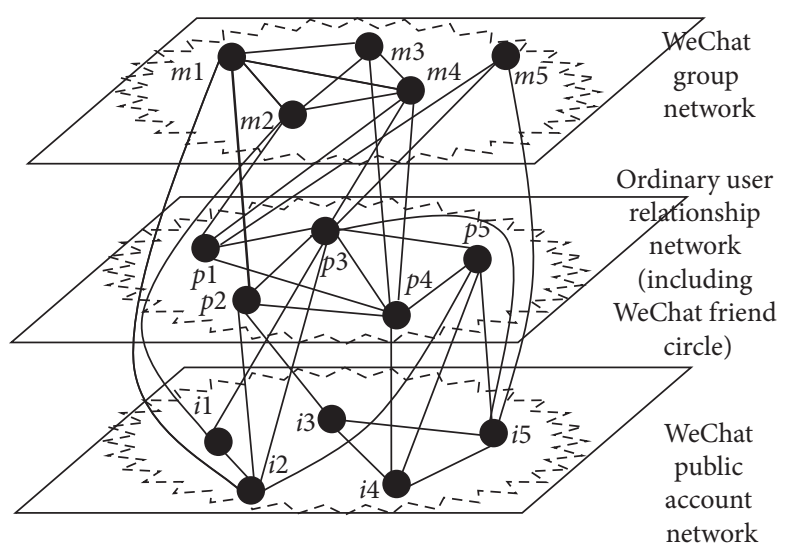

FIgURE 1: WeChat epidemic information dissemination Supernetwork.

are related, where the value 1 is time-dependent, and 0 is not relevant. Thus,

$$
\begin{aligned}
\text { WEIDSN } & =f\left(G_{i}, G_{p}, G_{m}\right) \\
& =G_{i}+G_{p}+G_{m}+E_{p-i}+E_{p-m}+E_{m-i} \\
& =\left(I, P, M, E_{I-I}, E_{P-P}, E_{M-M}, E_{p-i}, E_{p-m}, E_{m-i}\right),
\end{aligned}
$$

here, $E_{p-i}=\left\{\left(p_{i}, i_{j}\right) \mid \phi\left(p_{i}, i_{j}\right)=1\right\}$ represents a set of association relationships between $\mathrm{P}$ and I nodes; $E_{p-m}=$ $\left\{\left(p_{i}, m_{l}\right) \mid \varphi\left(p_{i}, m_{l}\right)=1\right\}$ represents a collection of association relationships between $\mathrm{P}$ and $\mathrm{M}$ nodes; and $E_{m-i}=$ $\left\{\left(i_{j}, m_{l}\right) \mid \gamma\left(i_{j}, m_{l}\right)=1\right\}$ represents a collection of associations between I and $M$ nodes.

It can be seen that the WeChat Supernetwork model is an integration of three epidemic information dissemination networks, which reveals the complex composition and structural form of epidemic information dissemination in WeChat.

\subsection{Construction of Adjacency Matrix and Network Intuitive} Graph of WeChat Epidemic Information Supernetwork Model. At this stage, we can use network analysis tools to measure various parameters of network analysis, and network relationship characterization software can draw network graphs for intuitive comparison.

Figure 1 is a schematic diagram of a three-network integration Supernetwork of a WeChat epidemic information dissemination system. The figure is divided into three layers. The top layer is the WeChat group network. The network members are WeChat groups. There may be associations between the network members, and the network members may also be associated with the members of the other two layers. $m_{i}$ represents the nodes in this layer network. The middle layer of the network is the ordinary WeChat user relationship network. The network members are ordinary WeChat users. There may be associations between the network members, and the network members may also be associated with members of the other two layers. $p_{i}$ represents nodes in this layer of the network. The bottom layer is the WeChat official account network. The network members are WeChat official accounts. There may be associations between the network members, and the network members may also be associated with members of the other two layers of networks. $i_{i}$ represents nodes in this layer network. Figure 1 is a schematic diagram of a WeChat epidemic information dissemination Supernetwork, in which ordinary WeChat user $p 2$ joins WeChat group $\mathrm{m} 1$ and ordinary WeChat users $p 3$ and $p 4$ join WeChat groups $m 4$ and $m 5$. After joining the WeChat groups $m 3$ and $m 4$, the ordinary WeChat user $p 1$ is added to the three WeChat groups $m 2, m 4$, and $m 5$ at the same time, and the ordinary WeChat user $p 5$ is temporarily not added to any WeChat group. WeChat groups $m 1, m 2$, and $m 5$ are associated with the public account network; ordinary WeChat user $p 2$ pays attention to the public accounts $i 2$ and $i 3$, ordinary user $p 3$ pays attention to the public accounts $i 1, i 2$, and $i 5$ at the same time, and ordinary user $p 4$ pays attention to the public account $i 4$, along with ordinary user $p 5$. After paying attention to the public accounts $i 2, i 4$, and $i 5$, the ordinary WeChat user $p 1$ does not follow any WeChat public account for the time being. Taking the WeChat epidemic information dissemination Supernetwork as an example, the boundary of the epidemic information dissemination subnetwork of the WeChat epidemic information dissemination system is determined, and its many network nodes and their associations are established. The rules for constructing the association relationship of network nodes are as follows. If the two nodes in the schematic diagram of the WeChat epidemic information dissemination Supernetwork are connected, they are marked as 1 ; otherwise, they are marked as 0 . Using network analysis software, the adjacency matrix of the WeChat epidemic information dissemination Supernetwork can be constructed as shown in Figure 2.

At the same time, the corresponding network analysis standard file can be generated and imported into the specified network relationship characterization software, which can be processed according to specific needs or purposes and used to then construct a network intuitive map of the WeChat epidemic information dissemination Supernetwork, as shown in Figure 3.

The representative $\mathrm{WeChat}$ epidemic information dissemination Supernetwork model outlined in this paper is a 


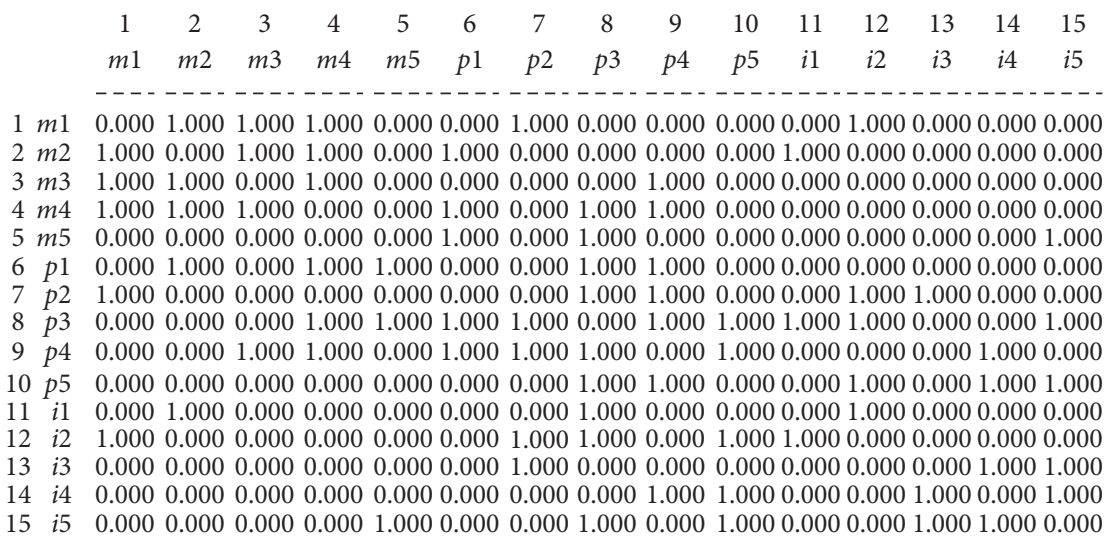

FIgURE 2: The adjacency matrix of the WeChat epidemic information Supernetwork.

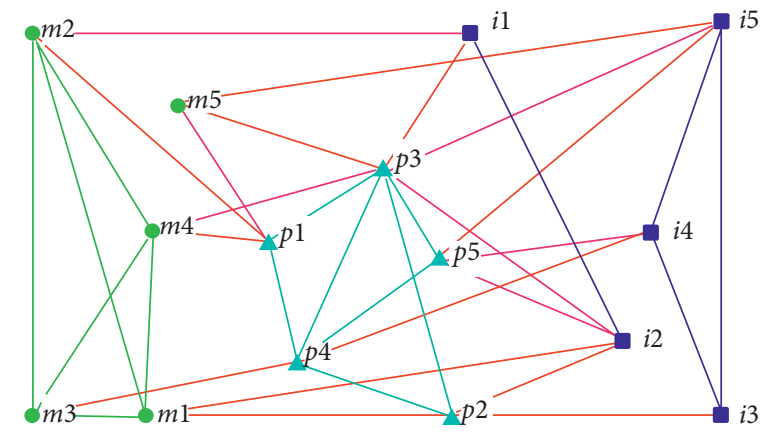

FIGURE 3: Intuitive map of the WeChat epidemic information dissemination Supernetwork.

network of three different types of epidemic information dissemination networks, including the public account network, WeChat group network, and common WeChat user relationship network. The Supernetwork model contains three relatively active and representative information carrier elements in the WeChat epidemic information dissemination system, such as WeChat public accounts, WeChat groups, common WeChat users, and their complex associations. Compared with the epidemic information dissemination network composed of similar nodes and their associations, the Supernetwork model can better reflect the complex composition and structural form of the WeChat epidemic information dissemination network system, so it can be more accurate, comprehensive and provide more indepth WeChat epidemic information. The communication network system conducts research and analysis, lays a foundation, and provides help for the management and guidance of subsequent WeChat epidemic information dissemination.

\section{Identification of Key Nodes in WeChat Epidemic Information Dissemination Supernetwork}

An important part of guiding the spread of epidemic-related information in WeChat is to first identify the key nodes in the spread of WeChat epidemic information. These important related nodes largely affect the spread of epidemic-related information in WeChat.

3.1. Traditional Key Node Identification Method. The degree centricity method in network analysis theory is the main method for identifying key nodes in a network. The formula is as follows:

$$
\begin{aligned}
& C_{D}\left(n_{i}\right)=d\left(n_{i}\right)=\sum_{j} X_{i j}=\sum_{j} X_{j i}, \\
& C_{D}^{\prime}\left(n_{i}\right)=\frac{d\left(n_{i}\right)}{g-1} .
\end{aligned}
$$

The value of $X_{j i}$ is 1 or 0 , indicating whether node $j$ is associated with node $i$, and $g$ is the number of nodes in the network. In the network, because the number of nodes in each network is different, and the number of large network nodes is large, the standardization process must be divided by the maximum possible number of relationships of a node in the network, that is, $g-1$ relationships $[19,20]$. Of course, in addition to the degree centrality method, there are many methods for identifying network central nodes, but the degree centrality method is relatively simple and straightforward, and the application scenarios of this method are also broader, with fewer restrictions on implementation [21]. The network analysis tool was used to calculate the degree centrality of all nodes in the Supernetwork, as shown in Figure 3, and the system calculation results are as follows. 
From the results in the above figure, we can see that the degree of centrality of node $p 3$ is 9 , the degree of centrality of $p 4$ is 7 , and the standardization of the two nodes is 0.64286 and 0.5 , respectively. It can be seen that the $p 3$ node and the $p 4$ node are relatively important. During the spread of WeChat epidemic-related information, monitoring and guiding these two nodes has a great impact on the overall information spread.

3.2. Key Node Recognition Method Based on WeChat Supernetwork. Degree centrality is currently one of the most commonly used key node identification methods, but its application in a Supernetwork has deficiencies. The degree centrality calculation here does not distinguish which subnet each node belongs to in the Supernetwork, there is no distinction between the relationships between nodes, and there is no difference between the relationships between subnets. Accordingly, this method cannot reflect the connection status of a node in the subnet to which it belongs, nor can it reflect the propagation ability of the node in the subnet to which it belongs; similarly, in the current situation, this method also fails to reflect the connection status of a node between subnetworks, and it fails to measure the propagation ability of the node between subnetworks. Referring to Figures 3 and 4, for example, the $i 1$ node has a degree of centrality of

$$
C_{D}(i 1)=d(i 1)=\sum_{j} X_{i j}=\sum_{j} X_{j i}=3 .
$$

And the $m 3$ node has a degree of centrality of

$$
C_{D}(m 3)=d(m 3)=\sum_{j} X_{i j}=\sum_{j} X_{j i}=4 \text {. }
$$

According to the formula,

$$
C_{D}^{\prime}\left(n_{i}\right)=\frac{d\left(n_{i}\right)}{g-1} .
$$

The standardized degree of centrality of $i 1$ can be obtained as

$$
C_{D}^{\prime}(i 1)=\frac{d(i 1)}{g-1}=0.21429 .
$$

And the standardized degree of centrality of node $m 3$ is

$$
C_{D}^{\prime}(m 3)=\frac{d(m 3)}{g-1}=0.28571 .
$$

Thus, node $i 1$ is not as important as $m 3$, but in the process of epidemic information propagation in WeChat, in the Supernetwork $i 1$ is associated with three unary subnets, while $m 3$ is only associated with the M-M network and P-P network and is not directly connected to the I-I network. From this perspective, $i 1$ is more important than $m 3$. The spread of epidemic information in WeChat has unique features. For example, the ability of ordinary WeChat users to spread information in the circle of friends is related to the number of their friends, the ability of the public account to spread information in WeChat is related to the number of fans, and the ability to spread information in WeChat groups and the number of WeChat group members is related. The nodes with strong propagation ability are more important, and the identification methods of these key nodes in WeChat need to be rebuilt.

In order to avoid the above deficiencies, this paper proposes a new comprehensive method for identifying key nodes in WeChat epidemic information dissemination based on a Supernetwork, part of which is based on an improvement of the decision method proposed previously [13], and the other part is mainly constructed based on the characteristics of the spread of epidemic information in WeChat. The new method is briefly described as follows.

Let $n_{i}$ be any node in the Supernetwork; $\mathrm{QC}_{D}\left(n_{i}\right)$ represents the degree of centrality of $n_{i}$ in the entire Supernetwork, and $\mathrm{ZC}_{D}\left(n_{i}\right)$ is the degree of centrality of $n_{i}$ in the unary subnet to which it belongs:

(1) Step 1. Calculate and compare the degree of centrality of each node in the entire Supernetwork.

(2) Step 2. Calculate and compare the degree centrality of all nodes in the M-P binary subnetwork and the I-P binary subnetwork in the M-P binary subnetwork and I-P binary subnetwork to which they belong.

(3) Step 3. Calculate and compare the degree centrality of all the individuals in the entire Supernetwork system in the subunit networks to which they belong.

(4) Step 4. Calculate and compare the internetwork propagation capabilities of each node in the subnet of a unitary network in the Supernetwork. Let LZS $\left(n_{i}\right)$ be the number of unitary subnetworks connected by node $n_{i}$ and ZJBS $\left(n_{i}\right)$ the edges of node $n_{i}$ between the unitary subnetworks. The number can be obtained from the above analysis. $\operatorname{ZJBS}\left(n_{i}\right)=\mathrm{QC}_{D}\left(n_{i}\right)-\mathrm{ZC}_{D}\left(n_{i}\right)$, the network propagation ability of the node $n_{i}$ in the Supernetwork is expressed by $\operatorname{ZJCN}\left(n_{i}\right)$, and the calculation and comparison methods are as follows:

$$
\begin{aligned}
& \text { Select Case } \operatorname{LZS}(A) \\
& \text { Case IS }>\operatorname{LZS}(B) \\
& \text { ZJCN }(A)>\operatorname{ZJCN}(B) \\
& \text { Case IS }<\operatorname{LZS}(B) \\
& \text { ZJCN }(A)<\operatorname{ZJCN~}(B) \\
& \text { Case IS }=\operatorname{LZS~}(B) \\
& \text { Select Case ZJBS }(A) \\
& \text { Case IS }>\text { ZJBS }(B) \\
& \text { ZJCN }(A)>\operatorname{ZJCN}(B) \\
& \text { Case IS }<\text { ZJBS }(B) \\
& \text { ZJCN }(A)<\text { ZJCN }(B) \\
& \text { Case IS }=\text { ZJBS }(B) \\
& \text { ZJCN }(A)=\text { ZJCN }(B) \\
& \text { End Select } \\
& \text { End Select }
\end{aligned}
$$




$\begin{array}{rcccc} & & 1 & 2 & 3 \\ & & \text { Degree } & \text { NrmDegree } & \text { Share } \\ & & ------ & ------ & ------ \\ 8 & p 3 & 9.000 & 64.286 & 0.122 \\ 9 & p 4 & 7.000 & 50.000 & 0.095 \\ 4 & m 4 & 6.000 & 42.857 & 0.081 \\ 10 & p 5 & 5.000 & 35.714 & 0.068 \\ 12 & i 2 & 5.000 & 35.714 & 0.068 \\ 6 & p 1 & 5.000 & 35.714 & 0.068 \\ 7 & p 2 & 5.000 & 35.714 & 0.068 \\ 1 & m 1 & 5.000 & 35.714 & 0.068 \\ 2 & m 2 & 5.000 & 35.714 & 0.068 \\ 15 & i 5 & 5.000 & 35.714 & 0.068 \\ 3 & m 3 & 4.000 & 28.571 & 0.054 \\ 14 & i 4 & 4.000 & 28.571 & 0.054 \\ 13 & i 3 & 3.000 & 21.429 & 0.041 \\ 11 & i 1 & 3.000 & 21.429 & 0.041 \\ 5 & m 5 & 3.000 & 21.429 & 0.041\end{array}$

FIGURE 4: The degree of centrality of all nodes in the WeChat epidemic information Supernetwork.

(5) Step 5. Calculate and compare the internetwork propagation capability $\mathrm{ZJCN}_{J}\left(n_{i}\right)$ between the M-type nodes in the M-P binary subnetwork and the I-type nodes in the I-P binary subnetwork under the corresponding binary subnetwork view. Let $J_{\mathrm{M}-\mathrm{P}} C_{D}\left(n_{i}\right)$ represent the degree of centrality of $n_{i}$ in the M-P binary subnetwork to which it belongs and let $J_{\mathrm{I}-\mathrm{P}} C_{D}\left(n_{i}\right)$ represent the degree of centrality of $n_{i}$ in the I-P binary subnetwork to which it belongs, and the calculation method of $\mathrm{ZJCN}_{J}\left(n_{i}\right)$ is as follows:

$$
\begin{aligned}
& \text { Select Case } n_{i} \\
& \text { Case IS } \in M \\
& \operatorname{ZJCN}_{J}\left(n_{i}\right)=J_{\mathrm{M}-\mathrm{P}} C_{D}\left(n_{i}\right)-\mathrm{ZC}_{D}\left(n_{i}\right) \\
& \text { Case IS } \in I \\
& \mathrm{ZJCN}_{J}\left(n_{i}\right)=J_{\mathrm{I}-\mathrm{P}} C_{D}\left(n_{i}\right)-\mathrm{ZC}_{D}\left(n_{i}\right) \\
& \text { End Select }
\end{aligned}
$$

(6) Combining the results of the above steps, key nodes are identified.

In the above steps, Step 2 prepares for the calculation and comparison of the number of fans of the public account, and the number of members in the WeChat group is determined in Step 5. Step 3, the calculation result, can also obtain the number of friends of ordinary WeChat users in the circle of friends. The circle of friends is one of the main channels for ordinary users to disseminate information in WeChat. The number of friends in the circle of friends is an important criterion for identifying the ability of ordinary WeChat users to disseminate information. Step 4 compares the unary subnet internetwork propagation capabilities of each node in the Supernetwork view by the number of unary subnetworks connected by the nodes and the number of nodes connected between the unary subnetworks. A ranking table was created showing the strengths and weaknesses of the unary subnet's internetwork spreading ability under the Supernetwork vision. Among them, $\mathrm{QC}_{D}\left(n_{i}\right)$ can be obtained by Step 1, $\mathrm{ZC}_{D}\left(n_{i}\right)$ can be obtained by Step 3 , and the individual's ZJBS $\left(n_{i}\right)$ can be obtained by the difference between $\mathrm{QC}_{D}\left(n_{i}\right)$ and $\mathrm{ZC}_{D}\left(n_{i}\right)$ of an individual. Firstly, it is judged based on the number of unary subnetworks connected to the node.
The number of unary subnetworks connected to node A is greater than the number of unary subnetworks connected to node $\mathrm{B}$, which indicates that the internetwork communication capability of node $A$ is stronger than that of node $B$. If the number of unary subnetworks connected to node $A$ is less than the number of unary subnetworks connected to node $\mathrm{B}$, it indicates that the internetwork propagation capability of node A between unary subnetworks is weaker than that of node B. If the number of unary subnetworks connected to node $\mathrm{A}$ is the same as the number of unary subnetworks connected to node $\mathrm{B}$, further judgments need to be made based on the number of edges between the unary subnetworks of nodes. Step 5 is mainly based on the calculation results of Step 2 and Step 3 and calculates and compares the number of fans of the public account and the number of members of the WeChat group in the WeChat Supernetwork. The information dissemination ability of nodes is measured to provide a reference for the identification of key nodes. This step calculates the internetwork propagation capability between the M-type nodes in the M-P binary subnetwork and the I-type nodes in the I-P binary subnetwork from the binary subnetwork perspective.

Taking the example shown in Figure 3 for verification and analysis:

(1) Step 1. The calculation result is shown in Figure 4.

(2) Step 2. The screenshots of the system calculation results are shown in Figures 5 and 6.

(3) Step 3. The result is shown in Figure 7.

(4) Step 4. The results are shown in Table 1.

(5) Combining the comparison and calculation results of Step 2 and Figures 5 and 6 with the comparison and calculation results of Step 3 and Figure 7, the calculation and comparison results of this step are shown in Tables 2 and 3.

(6) From the perspective of intranetwork propagation, Figure 7 shows that the degree of centrality of $i 3, i 4$, and $i 5$ in the I-I network is the same and the highest, and the propagation ability of $i 3, i 4$, and $i 5$ in the network is also the strongest. Nodes $m 1, m 2, m 3$, and 


$\begin{array}{ccccc} & & 1 & 2 & 3 \\ & & \text { Degree } & \text { NrmDegree } & \text { Share } \\ & & ------ & ------ & ------ \\ 3 & p 3 & 7.000 & 77.778 & 0.175 \\ 4 & p 4 & 5.000 & 55.556 & 0.125 \\ 5 & p 5 & 5.000 & 55.556 & 0.125 \\ 2 & p 2 & 4.000 & 44.444 & 0.100 \\ 7 & i 2 & 4.000 & 44.444 & 0.100 \\ 10 & i 5 & 4.000 & 44.444 & 0.100 \\ 9 & i 4 & 4.000 & 44.444 & 0.100 \\ 8 & i 3 & 3.000 & 33.333 & 0.075 \\ 1 & p 1 & 2.000 & 22.222 & 0.050 \\ 6 & i 1 & 2.000 & 22.222 & 0.050\end{array}$

Figure 5: The degree of centrality of all nodes in the I-P network.

$\begin{array}{rlccc} & & \begin{array}{c}1 \\ \text { Degree }\end{array} & \begin{array}{c}2 \\ \text { NrmDegree }\end{array} & \begin{array}{c}3 \\ \text { Share }\end{array} \\ & & ------ & ------ & ------ \\ 8 & p 3 & 6.000 & 66.667 & 0.143 \\ 4 & m 4 & 6.000 & 66.667 & 0.143 \\ 9 & p 4 & 6.000 & 66.667 & 0.143 \\ 6 & p 1 & 5.000 & 55.556 & 0.119 \\ 2 & m 2 & 4.000 & 44.444 & 0.095 \\ 1 & m 1 & 4.000 & 44.444 & 0.095 \\ 3 & m 3 & 4.000 & 44.444 & 0.095 \\ 7 & p 2 & 3.000 & 33.333 & 0.071 \\ 5 & m 5 & 2.000 & 22.222 & 0.048 \\ 10 & p 5 & 2.000 & 22.222 & 0.048\end{array}$

FIGURE 6: The degree of centrality of all nodes in the M-P network.

\begin{tabular}{|c|c|c|c|}
\hline & 1 & 2 & 3 \\
\hline & Degree & NrmDegree & Share \\
\hline & ------- & ------- & ------- \\
\hline $1 m 1$ & 3.000 & 75.000 & 0.250 \\
\hline $2 m 2$ & 3.000 & 75.000 & 0.250 \\
\hline $3 m 3$ & 3.000 & 75.000 & 0.250 \\
\hline $4 m 4$ & 3.000 & 75.000 & 0.250 \\
\hline $5 m 5$ & 0.000 & 0.000 & 0.000 \\
\hline & 1 & 2 & 3 \\
\hline & Degree & NrmDegree & Share \\
\hline & ------- & ------- & ------- \\
\hline $3 p 3$ & 4.000 & 100.000 & 0.286 \\
\hline $4 p 4$ & 4.000 & 100.000 & 0.286 \\
\hline $1 p 1$ & 2.000 & 50.000 & 0.143 \\
\hline $2 p 2$ & 2.000 & 50.000 & 0.143 \\
\hline $5 p 5$ & 2.000 & 50.000 & 0.143 \\
\hline & 1 & 2 & 3 \\
\hline & Degree & NrmDegree & Share \\
\hline & ------ & ------- & ------- \\
\hline $3 i 3$ & 2.000 & 50.000 & 0.250 \\
\hline $4 \quad i 4$ & 2.000 & 50.000 & 0.250 \\
\hline $5 i 5$ & 2.000 & 50.000 & 0.250 \\
\hline $2 i 2$ & 1.000 & 25.000 & 0.125 \\
\hline $1 i 1$ & 1.000 & 25.000 & 0.125 \\
\hline
\end{tabular}

FIgURE 7: The degree of centrality of each node in the unary subnet to which it belongs.

$m 4$ have strong propagation capabilities in the M-M network. In the P-P network, $p 3$ and $p 4$ are the strongest nodes in the network. From the perspective of internetwork propagation, Table 1 shows that the number of subnetworks connected by nodes $p 3$ and $i 2$ is larger, and the number of interconnected edges between networks is also larger, so their internetwork propagation capabilities are stronger. The degree centrality value of such nodes may not be large, but its influence on the propagation effect is often large, which is worthy of attention. It can also be seen from Table 1 that although $m 3$ has the strongest propagation capability in the M-M network, and $i 1$ has the weakest propagation capability in the I-I network in Figure 7, from the perspective of internetwork propagation, $i 1$ connects three subnets, there are 2 internetwork edges, $m 3$ only connects 2 subnets, and there is only 1 internetwork edge, so $i 1$ 
TABLE 1: Sequence table of node's internetwork epidemic information dissemination ability.

\begin{tabular}{lcc}
\hline Node & Number of unary subnets associated & Number of relationships \\
\hline$p 3$ & 3 & 5 \\
$i 2$ & 3 & 4 \\
$p 2$ & 3 & 3 \\
$p 4$ & 3 & 3 \\
$i 5$ & 3 & 3 \\
$m 1$ & 3 & 2 \\
$i 1$ & 3 & 2 \\
$m 2$ & 3 & 2 \\
$m 5$ & 2 & 3 \\
$p 1$ & 2 & 3 \\
$m 4$ & 2 & 3 \\
$p 5$ & 2 & 3 \\
$i 4$ & 2 & 2 \\
$i 3$ & 2 & 1 \\
\hline
\end{tabular}

TABLE 2: Sorting table of M-type nodes' epidemic information dissemination capability in WeChat.

\begin{tabular}{lccc}
\hline Node & $\begin{array}{c}\text { Degree of centrality } \\
\text { within the M-P network }\end{array}$ & $\begin{array}{c}\text { Degree centrality within } \\
\text { the M-M network }\end{array}$ & $\begin{array}{c}\text { Important indicators of WeChat } \\
\text { epidemic information dissemination capability }\end{array}$ \\
\hline$m 4$ & 6 & 3 & 3 \\
$m 5$ & 2 & 0 & 2 \\
$m 1$ & 4 & 3 & 1 \\
$m 2$ & 4 & 3 & 1 \\
$m 3$ & 4 & 3 & 1 \\
\hline
\end{tabular}

TABLE 3: Sorting table of the spreading capacity of epidemic information in WeChat for Class I nodes.

\begin{tabular}{lccc}
\hline Node & $\begin{array}{c}\text { Degree of centrality } \\
\text { within the I-P network }\end{array}$ & $\begin{array}{c}\text { Degree of centrality within } \\
\text { the I-I network }\end{array}$ & $\begin{array}{c}\text { Important indicators of WeChat } \\
\text { epidemic information dissemination capability }\end{array}$ \\
\hline$i 2$ & 4 & 1 & 3 \\
$i 4$ & 4 & 2 & 2 \\
$i 5$ & 4 & 2 & 2 \\
$i 3$ & 3 & 2 & 1 \\
$i 1$ & 2 & 1 & 1 \\
\hline
\end{tabular}

is slightly better than $m 3$ in terms of internetwork communication capability. From the perspective of the spread of epidemic-related information in WeChat, Figure 7 shows that $p 3$ and $p 4$ have a strong ability to spread epidemic-related information in the WeChat circle of friends. Under the same conditions, the spread of epidemic-related information of these two ordinary user nodes is faster. The spreading range is wide; Table 2 shows that $m 4$ has a strong spreading capability in WeChat group nodes. Under the same conditions, the information spreading speed of this WeChat group node is faster, and the spreading range is wider. Table 3 shows $i 2$ in the public account category. The dissemination capability of the node is strong. Under the same conditions, the public information node's epidemicrelated information spreads faster more widely. To guide the dissemination of epidemic-related information in WeChat, it is best to guide the abovementioned WeChat nodes with a strong ability to disseminate epidemic-related information.

The new method also identifies the importance of ordinary WeChat users, WeChat groups, and official accounts from the perspective of information diffusion based on the particularity of the spread of WeChat epidemic-related information, in order to be more comprehensive and reasonable.

\section{Conclusions}

This article mainly concerns the model construction and key node identification of the WeChat epidemic-related information dissemination Supernetwork. The identification method can be roughly divided into two parts. One part is based on the improvement of the decision method proposed previously [13], and the other part of it is mainly based on the characteristics of the spread of information related to the 
epidemic in WeChat. The Supernetwork model of WeChat epidemic information dissemination and the corresponding key node identification method constructed in this study are the initial attempts to research the information dissemination of epidemic-related information in WeChat.

\section{Data Availability}

The data used to support the findings of this study are included within the article.

\section{Conflicts of Interest}

The authors declare that they have no conflicts of interest regarding the publication of this paper.

\section{Authors' Contributions}

Peng Wu and Di Zhao contributed to the design of the study. Peng $\mathrm{Wu}$ gave the main idea of the manuscript, wrote the first draft, and performed the design of figures. All authors contributed to the manuscript revision and read and approved the submitted version.

\section{Acknowledgments}

This work was supported by the National Social Science Fund Project (18BGL238), China; the China Postdoctoral Science Foundation Special Funding Project (2018T110724), China; the Chinese Postdoctoral Science Fund General Support Project (2016M602236), China; and the Henan Philosophy and Social Science Planning Project (2017BJJ021); and Key Research Project of Teacher Education Curriculum Reform in Henan Province (2018-JSJYZD008), China.

\section{References}

[1] Science Net, "Mining social network opinion leaders," 2020, http://blog.sciencenet.cn/home.php? mod $=$ space $\& u i d=3075 \&$ do $=$ blog $\& i d=466991$.

[2] Surging News, "The 2019 WeChat annual data report is out!," 2020, https://www.thepaper.cn/newsDetail_forward_5480482.

[3] L. L. Liu, "Research on new coronary pneumonia epidemic reports from the perspective of the framework," Journalism Lover, vol. 5, pp. 17-21, 2020.

[4] Z. Deng, X. J. Liu, and Z. D. Kang, "Research on the public opinion propaganda and ideological education function of university new media in the prevention and control of major epidemics," Studies in Ideological Education, no. 3, pp. 38-43, 2020.

[5] Y. S. Chen, "Research on spreading and governance of internet rumors on public health emergency," E-Government, no. 6, pp. 2-11, 2020.

[6] B. J. Wan, C. Guo, Z. Zhao et al., "Characteristics and risks reflections of "anti-epidemic fitness messages" delivered by WeChat during the COVID-19 epidemic," Journal of Xi'an Physical Education University, vol. 37, no. 3, pp. 267-275, 2020.

[7] Y. Lu and L. Zhang, "Social media WeChat infers the development trend of COVID-19," Journal of Infection, no. 81, pp. 82-83, 2020.
[8] A. Nagurney and J. Dong, Supernetworks:Decision-Making for the Information Age, Edward Elgar Publishing, Cheltenham, UK, 2002.

[9] Z. P. Wang and Z. T. Wang, Supernetwork Theory and its Application, Science Press, Beijing, China, 2008.

[10] A. Nagurney, "Optimal supply chain network design and redesign at minimal total cost and with demand satisfaction," International Journal of Production Economics, vol. 128, no. 1, pp. 200-208, 2010.

[11] A. Nagurney, M. Yu, and Q. Qiang, "Supply chain network design for critical needs with outsourcing," Papers in Regional Science, vol. 90, no. 1, pp. 123-142, 2011.

[12] L. Zhu and J. Cao, "Supernetwork optimization of emergency resources allocation under disaster risk," Chinese Journal of Management Science, vol. 20, no. 6, pp. 141-148, 2012.

[13] P. Wu and H. S. Wang, "Modeling of information dissemination supernetwork in the emergencies and determination of the important nodes in the supernetwork," Journal of the China Society for Scientific and Technical Information, vol. 37, no. 7, pp. 722-729, 2012.

[14] Y. J. Xi, Y. Z. Dang, and K. J. Liao, "Knowledge supernetwork model and its application in organizational knowledge systems," Journal of Management Sciences in China, vol. 12, no. 3, pp. 12-21, 2009.

[15] P. Wu, H. S. Wang, Y. Li et al., "Judgment of important control nodes in the information dissemination super-network of emergency," Library and Information Service, vol. 57, no. 1, pp. 112-116, 2013.

[16] Y. Yu, Research on Knowledge Supernetwork in Organizational Knowledge Management, Dalian University of Technology, Dalian, China, 2009.

[17] J. L. Segovia-juarez, S. Colombano, and D. Kirschner, "Identifying DNA splice sites using hypernetworks with artificial molecular evolution," Biosystems, vol. 87, no. 2-3, pp. 117-124, 2007.

[18] Z. T. Wang, "Reflection on supernetwork," Journal of University of Shanghai for Science and Technology, vol. 33, no. 3, pp. 229-237, 2011.

[19] J.-B. Liu, J. Cao, A. Alofi, A. AL-Mazrooei, and A. Elaiw, "Applications of laplacian spectra for n-prism networks," Neurocomputing, vol. 198, pp. 69-73, 2016.

[20] J. D. Luo, Lecture Notes on Social Network Analysis, Social Sciences Academic Press, Beijing, China, 2nd edition, 2010.

[21] S. Hayat, S. Wang, and J.-B. Liu, "Valency-based topological descriptors of chemical networks and their applications," Applied Mathematical Modelling, vol. 60, pp. 164-178, 2018. 